\title{
Prevalence and Biomarkers of Diabetic Kidney Disease in Diabetic Patients on Treatment in Buea and Ngaoundere, Cameroon
}

\author{
Mbarawa Marat Kofia Ibrahim ${ }^{1,2}$, Asongalem Emmanuel Acha ${ }^{3}$, Nsagha Dickson Shey ${ }^{4}$, \\ Njouendou Abdel Jelil ${ }^{2,3}$, Assob Nguedia Jules Clement ${ }^{2,3, *}$ \\ ${ }^{1}$ Department of Medical Laboratory Sciences, Faculty of Health Sciences, University of Buea, Buea, Cameroon \\ ${ }^{2}$ Medical Research and Applied Biochemistry Laboratory, Faculty of Health Sciences, University of Buea, Buea, Cameroon \\ ${ }^{3}$ Department of Biomedical Sciences, Faculty of Health Sciences, University of Buea, Buea, Cameroon \\ ${ }^{4}$ Department of Public Health and Hygiene, Faculty of Health Sciences, University of Buea, Buea, Cameroon
}

Email address:

mbarawamarat@gmail.com (M. M. K. Ibrahim),cpehw@yahoo.com (A. E. Acha),nsaghads@hotmail.com (N. D. Shey), ajnjouendou@gmail.com (N. A. Jelil),juleclement@gmail.com (A. N. J. Clement)

${ }^{*}$ Corresponding author

\section{To cite this article:}

Mbarawa Marat Kofia Ibrahim, Asongalem Emmanuel Acha, Nsagha Dickson Shey, Njouendou Abdel Jelil, Assob Nguedia Jules Clement. Prevalence and Biomarkers of Diabetic Kidney Disease in Diabetic Patients on Treatment in Buea and Ngaoundere, Cameroon. American Journal of Bioscience and Bioengineering. Vol. 8, No. 5, 2020, pp. 84-91. doi: 10.11648/j.bio.20200805.11

Received: July 23, 2020; Accepted: August 4, 2020; Published: October 30, 2020

\begin{abstract}
Diabetic Kidney Disease (DKD) is a complication of diabetes that often leads to the End Stage Renal Disease. It is characterised by the presence of persistent albuminuria and a reduction of the Glomerular Filtration Rate (GFR) in diabetic condition. No study has revealed the prevalence of DKD in Cameroon. This cross-sectional study was conducted in Buea and Ngaoundere to determine the prevalence of DKD and characterize its biochemical profile in diabetic population under medical care. A total of 250 diabetics were enrolled with a mean age of $56.78 \pm 12.06$ years, out of which $59.6 \%$ were diagnosed with Chronic Kidney Disease (CKD), 32.8\% presented micro-albuminuria and 3.6\% were diagnosed with macroalbuminuria. The prevalence of DKD was $15.2 \%$ out of which $78.9 \%$ were females $(\mathrm{p}=0.002)$. The prevalence of the co-morbidity hypertension and DKD was $8.8 \%$. Significant association was found between DKD and two variables: female gender (OR: 2.28 (1.21-4.29); $\mathrm{p}=0.002)$ and hyper-creatinemia (OR: $3.47(2.13-5.66) ; \mathrm{p}<0.001)$. The high prevalence of micro-albuminuria found in this study may reflect a high frequency of micro-albuminuria in diabetic population in Cameroon. This study is the first, to assess DKD in Cameroon according to the ADA consensus on CKD and diabetes. The findings showed that, diabetic complication is a serious problem in Cameroon and, more actions should be taken to improve its management.
\end{abstract}

Keywords: DKD, Diabetes, Cameroon, GFR, ADA Consensus, KDIGO

\section{Introduction}

Diabetic Kidney Disease (DKD), a complication that occurs in about 20 to $40 \%$ of diabetic population [1] can progress to the End Stage of Renal Disease. It is more frequent in African-American individuals and, has been identified as one of the main causes of mortality due to complication of diabetes worldwide. DKD occurs in type 1 and type 2 diabetic patients with poor glycaemic control [2]. The pathophysiology of DKD occurs in the glomerulus by the expansion of mesangial, the thickening of the tubular, the loss of the endothelial fenestration and podocytes, the development of Kimmelstiel-Wilson nodules and, the appearance of the glomerular sclerosis [3]. Recent experimentations have shown the influences of inflammatory factors in the structural changes of glomerulus [4]. Tumour Necrosis Factor and Reactive Oxygen Species, activators of the nuclear factor kappa-light-chain-enhancer, the regulator of inflammatory factors, are associated with proteinuria. The persistence of immune response participates highly in the acceleration of kidney structure changes and the decline of 
renal function in diabetic condition. Clinically, DKD is defined by the presence of persistent albuminuria, added to a reduction of the Glomerular Filtration Rate (GFR) in diabetic condition. It is due to the haemodynamic dysfunction in the kidney, which has as consequence, the reduction of the vascular resistance in both afferent and efferent arterioles leading to intra-renal hydrostatic pressure, then, the disruption in the autoregulation of its system [2]. The presence of kidney dysfunctions in diabetic condition doesn't automatically indicate DKD. In type 1 diabetes, the histological changes are frequently seen in DKD condition but, in type 2, these could indicate other pathogenic conditions. Then, it is some time difficult to diagnose true DKD, Non-diabetic Kidney Disease or the co-existence of the two forms [4]. Despite the confusion within these diseases, a consensus has been achieved by the American Diabetes Association (ADA), the American Society of Nephrology (ASN) and the National Kidney Foundation (NKF) to validate an approach for the identification, diagnostic and management of DKD. Thus, the ADA consensus conference on $\mathrm{CKD}$ and diabetes recommended the diagnosis of DKD using laboratory assessment by the identification of the estimated GFR at a level less than 60 $\mathrm{ml} / \mathrm{min} / 1.73 \mathrm{~m} 2$ and, the presence of kidney damages, estimated by albuminuria greater or equal to $30 \mathrm{mg} / \mathrm{g}$ of creatinine [5]. This recommendation has facilitated the clinical identification of DKD and helped in the understanding of the epidemiology of this disease. Nowadays, the worldwide prevalence of DKD has not been fully estimated, however, data from studies carried out in countries or limited regions are available. In the USA in 2008, the prevalence of DKD was $3.3 \%$ in the general population and $34.5 \%$ in diabetic population [6]. This prevalence was $10.8 \%$ in the diabetic population in Saud Arabia [7] and 6.7\% in Egypt [8]. However, no study has been carried out in Cameroon using the above mentioned ADA consensus recommendations for the identification of DKD cases [5]. This present study was therefore conducted to estimate the prevalence of DKD in diabetic population and, to characterize their biochemical profile.

\section{Material and Methods}

\subsection{Study Site and Design}

This cross-sectional and multi-centric study was conducted from January 2018 to April 2019 in Buea and Ngaoundere, the headquarters of South-West and Adamawa Regions of Cameroon respectively. In Buea, participants were recruited from the Buea Regional Hospital and the Lambe Diabetic Foundation of Buea while in Ngaoundere, they were from the Ngaoundere Regional Hospital.

\subsection{Study Population and Recruitment Strategy}

This study targeted individuals that were diagnosed diabetics in the 3 selected health facilities. Using a random sampling technique, we included patients who were aged 18 years and above, diagnosed with diabetes and who accepted to sign the consent form. Menstruating females were asked to return after their menstrual period. Individuals who performed intensive physical exercises within the previous 72 hours were also asked to return after 72 hours period after resting. During the study, kidney functions of participants were assessed. For individuals with reduced kidney function according to our method, another appointment was established after 3 months period for a second assessment.

\subsection{Data Collection and Management}

Socio-demographic and clinical data were collected using a questionnaire. During each visit, we collected $3 \mathrm{ml}$ of fresh blood sample from each participant in a dried tube. The same day of the recruitment, patients received a clean, dried and sterile container with no preservative for urine collection. They were prior trained on how to collect the first morning urine, and bring on the next day to health facility. Blood and urine samples that were not analysed during the day of collection were stored in a freezer at $2-4^{\circ} \mathrm{C}$ for analysis within the next 24 hours, or in another big freezer at $-70^{\circ} \mathrm{C}$ in the case of long term storage in cryo-tubes.

\subsection{Biochemical Analysis}

Biomarkers of kidney function were measured using the semi-automated spectrophotometer biochemistry analyser, MCL-302B. Fasting blood glucose (FBG), serum creatinine, urea, Low Density Lipoprotein (LDL) cholesterol, High Density Lipoprotein (HDL) cholesterol, total cholesterol and Triglyceride (TG) as well as urine creatinine and albumin were analysed using specific reagent kits. The kits used to measure FB, Creatinine, Urea and Albumin were purchased from Chronolab Company in Barcelona, Spain. Kits for LDL, HDL and TG were procured from Biolabo Company in Maizy, France.

\subsection{Assessment of Albumin-Creatinine Ration (ACR)}

Albuminuria was assessed by determining ACR in urine. Albuminuria was normal when the result of ACR was less than $30 \mathrm{mg} / \mathrm{g}$. Values between 30 and 299 indicated microalbuminuria. Results greater or equal to $300 \mathrm{mg} / \mathrm{g}$ were the indication of macro-albuminuria.

\subsection{Estimation of the GFR}

The estimation of GFR was done using MDRD formula developed by Levey et Al. [9]. This formula has been taken in consideration in accordance with the indication of Levey where MDRD has been advised for people with diabetes.

\subsection{Diagnostic of $\mathrm{DKD}$}

The diagnostic of DKD was done according to the recommendations from the consensus of ADA, ASN and NFK during the 2014 conference on CKD and Diabetes. There, DKD was considered present when eGFR repeated 
twice, after 3 months interval period at least, was less than 60 $\mathrm{ml} / \mathrm{min} / 1.73 \mathrm{~m} 2$ with micro-albuminuria or, persistent macroalbuminuria. We considered the presence of retinopathy coupled to micro-albuminuria as a marker of DKD in diabetic condition, without any other cause of CKD [5].

\section{Ethical Consideration}

The research protocol was approved by the Institutional Ethics Committee for Research on Human Health of the University of Douala. An Ethical clearance No 1157 IEC$\mathrm{UD} / 11 / 2017 / \mathrm{T}$ was granted for the study. Administrative clearance was obtained from the South-West and Adamawa Regional Delegations of Public Health. The Regional Hospital of Buea, the Regional Hospital of Ngaoundere and the Lambe Foundation of Diabetes of Buea, granted each an administrative authorization to carry-out this research.

\section{Statistical Analysis}

Data were entered into a spreadsheet of Microsoft Office Excel 2016 and analysed using SPSS 22.0. Descriptive statistics of variables were expressed as mean \pm standard deviation (SD), frequencies and percentages. Chi-square or Fisher's exact tests were used to assess association between variables with $95 \%$ Confidence Intervals (CI). Student-t test was used to compare means of variables. Risk factors were assessed with Odds ratios (OR) and 95\% CI. $P$-values $<0.05$ were considered statistically significant.

Table 1. Socio-demographic characteristic of the population in Buea and Ngaoundere centers.

\begin{tabular}{llll}
\hline Variable & Buea N (\%) & Ngaoundere N (\%) & Total N (\%) \\
\hline Sex & & & \\
$\begin{array}{l}\text { Female } \\
\text { Male }\end{array}$ & $58(23.2)$ & $52(20.8)$ & $110(44)$ \\
$\begin{array}{l}\text { Marital status } \\
\quad \text { Single }\end{array}$ & $72(28.8)$ & $68(27.2)$ & $140(56)$ \\
$\quad$ Married & $23(09.2)$ & $17(06.8)$ & $40(21.0)$ \\
$\quad$ Divorced & $16(16.8)$ & $62(24.8)$ & $104(41.6)$ \\
\hline
\end{tabular}

\begin{tabular}{clll}
\hline Variable & Buea N (\%) & Ngaoundere N (\%) & Total N (\%) \\
\hline $\begin{array}{c}\text { Widow (er) } \\
\text { Religion }\end{array}$ & $49(19.6)$ & $36(14.4)$ & $85(34.0)$ \\
Islam & $10(04.0)$ & $78(31.2)$ & $88(35.2)$ \\
Christianism & $106(42.4)$ & $39(15.6)$ & $145(58.0)$ \\
Others & $14(05.6)$ & $3(01.2)$ & $17(06.8)$ \\
Residence & & & \\
Urban & $117(46.8)$ & $109(43.6)$ & $226(90.4)$ \\
Rural & $13(05.2)$ & $11(04.4)$ & $24(09.6)$ \\
Age group (year) & & & $8(3.2)$ \\
$\leq 35$ & $6(2.4)$ & $2(00.8)$ & $50(20.0)$ \\
$36-45$ & $21(08.4)$ & $29(11.6)$ & $47(18.8)$ \\
$46-55$ & $26(10.4)$ & $21(08.4)$ & $76(30.4)$ \\
$56-65$ & $35(14.0)$ & $41(16.4)$ & $57(22.8)$ \\
$66-75$ & $37(14.8)$ & $20(08.0)$ & $12(4.8)$ \\
$\geq 76$ & $5(02.0)$ & $7(02.8)$ & $250(100.0)$ \\
\hline Overall & $130(52.0)$ & $120(48.0)$ & \\
\hline
\end{tabular}

N: Frequency. \%: Percentage.

\section{Results}

\subsection{Demographic and Clinical Characteristics of Participants}

During this study, we met 275 participants and excluded 25 after carefully cheeking according to our criteria and guidelines, explained above. The final number of participants enrolled was 50 . As shown in table 1 above, the mean age was $56.78 \pm 12.06$ years with a minimum of 28 years and a maximum of 89 years. The Clinical characteristics are presented in table 2 below. It can be seen that, the female population represented $56.0 \%$ with a mean's age of 56.2 \pm 10.8 years (CI: $54.4-57.8$ ) while the male population had an average of $57.6 \pm 13.3$ years (CI: $55.3-60.0)$. Obesity was prevalent at $63 \%$, including pre-obesity and obesities of class I, II and III, with predominance in female population $(\mathrm{P}=0.043)$. The age group 56-65 years was the more present (30.4\%) and, more than half of the population (50.4\%) had duration of diabetes less than 5 years. The prevalence of hypertension was $60.4 \%$ while, within sex groups, it was higher in males compared to females $63.6 \%$ and $57.8 \%(\mathrm{P}=0.585)$ respectively.

Table 2. Clinical characteristic of diabetics patients according to the gender.

\begin{tabular}{|c|c|c|c|c|c|}
\hline \multirow{2}{*}{ Clinical variables } & \multirow{2}{*}{ Female N (\%) } & \multirow{2}{*}{ Male N (\%) } & \multirow{2}{*}{ Total N (\%) } & \multicolumn{2}{|c|}{ Statistics } \\
\hline & & & & $\mathbf{X}^{2}$ & $P$-value \\
\hline \multicolumn{6}{|l|}{ Diagnosed hypertensive } \\
\hline Yes & $81(32.4)$ & $70(28.0)$ & $151(60.4)$ & 1.07 & 0.585 \\
\hline Sub-total of diagnosed hypertensive & $140(56.0)$ & $110(44.0)$ & $250(100.0)$ & & \\
\hline \multicolumn{6}{|l|}{ BMI } \\
\hline Normal weight & $39(15.7)$ & $49(19.7)$ & $88(35.3)$ & \multirow{5}{*}{11.44} & \multirow{5}{*}{$0.043 *$} \\
\hline Pre-obesity & $68(27.3)$ & $40(16.1)$ & $108(43.4)$ & & \\
\hline Obesity class I & $14(5.6)$ & $14(5.6)$ & $28(11.2)$ & & \\
\hline Obesity class II & $10(4.0)$ & $4(1.6)$ & $14(5.6)$ & & \\
\hline Obesity class III & $5(2.0)$ & $2(0.8)$ & $7(2.8)$ & & \\
\hline Sub-total BMI & $140(56.0)$ & $110(44.0)$ & $250(100.0)$ & & \\
\hline$\leq 5$ Years & $77(30.8)$ & $49(19.6)$ & $126(50.4)$ & \multirow[t]{2}{*}{3.31} & \multirow[t]{2}{*}{0.190} \\
\hline 6 to 10 Years & $37(14.8)$ & $40(16.0)$ & $77(30.8)$ & & \\
\hline
\end{tabular}




\begin{tabular}{clllll}
\hline Clinical variables & Female N (\%) & Male N (\%) & Total N (\%) & Statistics \\
\cline { 4 - 5 } & & & $\mathbf{X}^{2}$ & \multicolumn{1}{c}{$\boldsymbol{P}$-value } \\
\hline Sub-total duration diabetes & $26(10.4)$ & $21(8.4)$ & $47(18.8)$ & \\
\hline
\end{tabular}

N: Frequency. \%: Percentage. BMI: Body Mass Index. $X^{2}$ : Chi Square test. df: Degree of Freedom.

\subsection{Biomarkers of DKD in Diabetic Study Population}

The mean of FBG was $164.56 \pm 72.90 \mathrm{mg} / \mathrm{dL}$ and females presented a higher value compared to males, with respectively $168.33 \pm 74.46 \mathrm{mg} / \mathrm{dL}$ (CI: 155.59-171.57) and $160.42 \pm 71.01 \mathrm{mg} / \mathrm{dL}$ (CI: 147.87-174.68). The mean value of serum creatinine was $0.94 \pm 0.32 \mathrm{mg} / \mathrm{dL}$, for the entire population of study. During the evaluation of the main lipid markers, we found that Total Cholesterol was equal to $206.11 \pm 66.89 \mathrm{mg} / \mathrm{dL}$, HDL Cholesterol equal to $66.67 \pm 46.54$ $\mathrm{mg} / \mathrm{dL}$ and $\mathrm{TG}$ equal to $177.18 \pm 76.56 \mathrm{mg} / \mathrm{dL}$. LDL Cholesterol was at $103.7 \pm 73.32 \mathrm{mg} / \mathrm{dL}$. According to the duration of diabetes, people with a duration greater than 5 years presented higher values of FBG, serum urea and serum HDL compared to those with duration of less or equal to 5 years. In another hand, serum creatinine, Total cholesterol, TG and LDL Cholesterol were elevated in people with duration of less or equal to 5 years, compared to those with duration of greater than 5 years (Table 3 ).

Table 3. Description of biomarkers' means according to the duration of diabetes.

\begin{tabular}{llllll}
\hline \multirow{2}{*}{ Variable } & \multicolumn{2}{l}{ People with diabetes $\leq \mathbf{5}$ years } & \multicolumn{2}{c}{ People with diabetes > 5 years } & Statistics \\
\cline { 2 - 6 } & Mean & SD & Mean & SD & t-test \\
\hline FBG (mg/dL) & 160.04 & 67.55 & 169.16 & 77.96 & 0.989 \\
S. Creat (mg/dL) & 0.951 & 0.3403 & 0.932 & 0.3091 & 0.481 \\
S. BUN (mg/dL) & 44.61 & 42.604 & 41.20 & 62.861 & 0.502 \\
S. Urea (mg/dL) & 65.77 & 56.657 & 70.96 & 67.050 & 0.661 \\
TC (mg/dL) & 209.37 & 63.618 & 202.79 & 70.179 & 0.777 \\
TG (mg/dL) & 180.69 & 72.653 & 173.62 & 80.476 & 0.61 \\
HDL (mg/dL) & 63.42 & 44.410 & 70.58 & 48.532 & 0.509 \\
LDL (mg/dL) & 109.81 & 77.763 & 97.49 & 68.271 & 0.438 \\
\hline
\end{tabular}

FBG: Fasting Blood Glucose. S. Creat: Serum Creatinine. S. Urea: Serum Urea. BUN: Blood Urea Nitrogen. TC: Total Cholesterol. TG: Triglycerides. HDL: High Density Lipoprotein. LDL: Low Density Lipoprotein. SD: Standard Deviation. df: Degree of freedom.

\subsection{Assessment of CKD in the Study Population}

The assessment of kidney function, according to the estimation of GFR, shown general mean of the population at $100.57 \mathrm{ml} / \mathrm{min} / 1.73 \mathrm{~m} 2$ where male population presented a more elevated value compared to female population with respectively $112.00 \pm 47.11 \mathrm{ml} / \mathrm{min} / 1.73 \mathrm{~m}^{2}$ (CI: $103.23-120.24$ ) and $91.54 \pm 55.46 \mathrm{ml} / \mathrm{min} / 1.73 \mathrm{~m} 2$ (CI: 83.43-102.40). Microalbuminuria was found in $32.8 \%$ of participants and $3.6 \%$ were diagnosed with macro-albuminuria (figure 1). According to the classification of $\mathrm{CKD}$ as recommended by KDIGO, using the MDRD formula, $59.6 \%$ of participants presented CKD. The different stages, 1, 2, 3a, 3b, 4 and 5 were counted with the respective cases of $28(11.2 \%), 83(33.2 \%), 33(13.2 \%), 3$ (1.2\%), $2(0.8 \%)$ and $0(0.0 \%)$ (Figure 2$)$.

Out of the 250 participants, 38 presented DKD which represented a prevalence of $15.2 \%$ where, $78.9 \%$ were females $(\mathrm{P}=0.002)$ (Table 4). The prevalence of the comorbidity hypertension and DKD was $8.8 \%(\mathrm{P}=0.732)$ and, $9.2 \%$ were people diagnosed DKD with diabetes duration of less than 5 years $(\mathrm{P}=0.175)$. Within the diagnosed $\mathrm{DKD}$ patients group, the prevalence of hypertension was found to be $28.9 \%$. The mean FBG of people diagnosed DKD was lower than those without $\mathrm{DKD}(\mathrm{P}=0.666)$ while serum creatinine was higher in people diagnosed DKD compared to those without DKD $(\mathrm{P}<0.001)$. Total Cholesterol $(\mathrm{P}=0.900)$, TG $(\mathrm{P}=0.923)$ and LDL Cholesterol $(\mathrm{P}=0.551)$ were elevated in people with DKD compared to those without DKD (Table 5). Associations were assessed between the presence of DKD and some variables using a multivariate analysis. Significant association was found between DKD and two variables, female gender $(\mathrm{P}=0.002)$ and hyper-creatininemia $(\mathrm{P}<0.001)$, with respectively $\mathrm{OR}$ of 2.28 (CI: $1.21-4.29)$ and 3.47 (CI: 2.13-5.66) (Table 6).

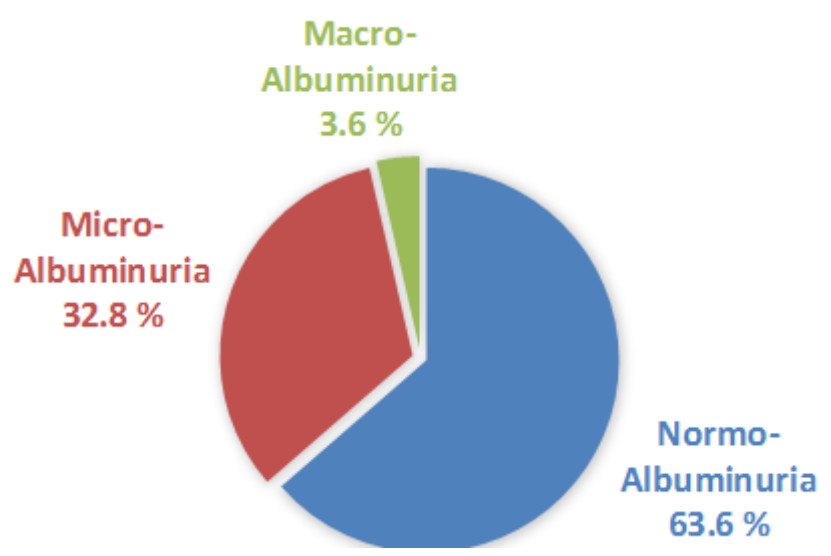

Figure 1. Prevalence of micro- and macro-albuminuria in the study population. 


\subsection{Prevalence and risk Factors of DKD in the Study Population}

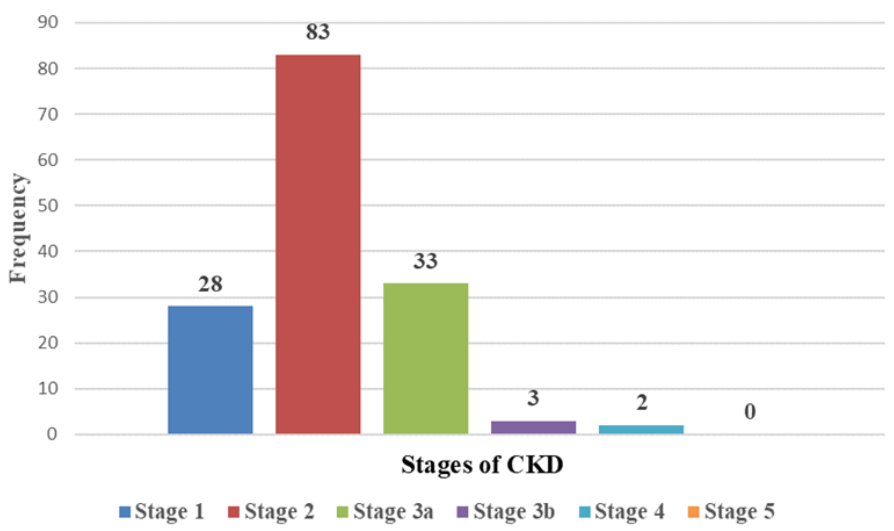

Figure 2. Frequencies of different stages of CKD in the study population.

Table 4. Comparison of biochemical parameter's mean according to DKD status.

\begin{tabular}{|c|c|c|c|c|c|c|c|}
\hline \multirow{2}{*}{ Variable } & \multicolumn{2}{|c|}{ People without DKD } & \multicolumn{2}{|c|}{ People with DKD } & \multicolumn{3}{|c|}{ Statistics } \\
\hline & Mean & SD & Mean & SD & T-test & df & P-value \\
\hline FBG (mg/dL) & 165.53 & 70.59 & 160.28 & 83.07 & 0.44 & 248 & 0.666 \\
\hline S. Creat $+(\mathrm{mg} / \mathrm{dL})$ & 0.85 & 0.24 & 1.33 & 0.34 & -10.89 & 248 & $<0.001 *$ \\
\hline S. BUN (mg/dL) & 44.53 & 58.24 & 35.76 & 22.20 & 1.00 & 248 & 0.317 \\
\hline S. Urea $(\mathrm{mg} / \mathrm{dL})$ & 68.74 & 64.49 & 66.59 & 49.72 & 0.21 & 248 & 0.832 \\
\hline $\mathrm{TC}(\mathrm{mg} / \mathrm{dL})$ & 205.85 & 65.95 & 207.23 & 71.68 & -0.12 & 248 & 0.900 \\
\hline $\mathrm{TG}(\mathrm{mg} / \mathrm{dL})$ & 176.96 & 75.81 & 178.17 & 80.65 & -0.09 & 248 & 0.923 \\
\hline $\mathrm{HDL}(\mathrm{mg} / \mathrm{dL})$ & 68.08 & 49.06 & 62.06 & 33.16 & 1.00 & 95 & 0.317 \\
\hline LDL (mg/dL) & 102.38 & 72.64 & 109.53 & 76.80 & -0.57 & 248 & 0.551 \\
\hline
\end{tabular}

FBG: Fasting Blood Glucose. S. Creat: Serum Creatinin. S. Urea: Serum Urea. BUN: Blood Urea Nitrogen. TC: Total Cholesterol. TG: Triglycerides. HDL: High Density Lipoprotein. LDL: Low Density Lipoprotein. SD: Standard Deviation. df: Degre of freedom. *: $P$-value $<0.05$.

Table 5. Characteristic of the population diagnosed with DKD.

\begin{tabular}{|c|c|c|c|c|}
\hline Variable & & People with DKD N (\%) & People without DKD N (\%) & P-value \\
\hline \multicolumn{5}{|l|}{ Sex } \\
\hline & Female & $30(12.0)$ & $110(44.0)$ & $0.001 *$ \\
\hline & Male & $8(3.2)$ & $102(40.8)$ & \\
\hline Sub-total & & $38(15.2)$ & $212(84.8)$ & \\
\hline \multicolumn{5}{|c|}{ Diagnosed hypertensive } \\
\hline & No & $16(6.4)$ & $83(33.2)$ & 0.432 \\
\hline & Yes & $22(8.8)$ & $129(51.6)$ & \\
\hline Sub-total & & $38(15.2)$ & $212(84.8)$ & \\
\hline \multicolumn{5}{|c|}{ Diagnosed DD } \\
\hline & No & $27(10.8)$ & $160(64.0)$ & 0.347 \\
\hline & Yes & $11(4.4)$ & $52(20.8)$ & \\
\hline Sub-total & & $38(15.2)$ & $212(84.8)$ & \\
\hline \multicolumn{5}{|c|}{ Duration of diabetes } \\
\hline & $\leq 5$ Years & $23(9.2)$ & $103(41.2)$ & 0.119 \\
\hline & $>5$ Years & $15(6.0)$ & $109(43.6)$ & \\
\hline Sub-total & & $38(15.2)$ & $212(84.8)$ & \\
\hline
\end{tabular}

N: Frequency. \%: Percentage. DD: Diabetic Dyslipidemia, DKD: Diabetic Kidney Disease. *: $p$-value $<0.05$ (Fisher's Exact test)

Table 6. Multivariate analysis of possible risk factors for DKD.

\begin{tabular}{lll}
\hline Variable & Adjusted OR (95\% CI) & p-value \\
\hline Female Sex & $2.28(1.21-4.29)$ & $0.002^{* *}$ \\
Diagnosed hypertensive & $1.05(0.78-1.40)$ & 0.760 \\
Diagnosed DD & $1.06(0.85-1.32)$ & 0.548 \\
Duration of diabetes less than 5 years & $1.30(0.86-1.97)$ & 0.119 \\
Hyper-glycaemia & $1.09(0.83-1.43)$ & 0.511 \\
Hyper-Creatininemia & $3.47(2.13-5.66)$ & $<0.001^{* *}$ \\
Hyper-Total Cholesterolemia & $1.07(0.79-1.44)$ & 0.648 \\
Hyper-LDL Cholesterolemia & $1.03(0.84-1.25)$ & 0.751 \\
Hyper-HDL Cholesterolemia & $1.14(0.74-1.75)$ & 0.536 \\
\hline
\end{tabular}




\begin{tabular}{lll}
\hline Variable & Adjusted OR (95\% CI) & $\boldsymbol{p}$-value \\
\hline Hyper- Triglyceridemia & $1.12(0.87-1.44)$ & 0.311 \\
\hline
\end{tabular}

LDL: Low Density Lipoprotein. HDL: High Density Lipoprotein. DKD: Diabetic Kidney Disease. DD: Diabetic Dyslipidemia. CI: Confidence of interval OR: Odd Ratio. CI: Confidence of Interval.. **: P-value $<0.05$.

\section{Discussion}

This present research has been done in diabetic people living in Buea and Ngaoundere, two regional headquarters of Cameroon, in Central Africa, to determine the prevalence of DKD and to describe some biomarkers related to this disease. The research has involved 250 participants from 3 selected health facilities. The assessment of DKD has followed the crucial recommendations of ADA consensus on diabetes and CKD [5].

From our study, we found that the prevalence of DKD is $15.2 \%$. Prevalences of $6.7 \%, 10.3 \%$ and $34.5 \%$ have been found respectively in diabetic populations in Egypt [8], in the Kingdom of Saud Arabia (KSA) [6] and in the United States of America (USA). The heterogeneity in the prevalence found in our study with those countries is probably due to the ethnicity and racial influences associated to the development of DKD. Although studies suggested that, black peoples are more exposed developing DKD compared to others [11, 12]. The higher prevalence of DKD in USA compared to our study may be due to the complex socio-ethnoracial composition of this population and their lifestyle. The high prevalence of obesity and hypertension in the USA population with diabetes, which are respectively $85.2 \%$ [13] and $73.6 \%$ [14], added to metabolic syndrome and the American lifestyle, compared to the findings in our study population, with prevalence of obesity at $60.0 \%$ and hypertension at $60.4 \%$, may be responsible to the difference between the two DKD prevalence's. The prevalence of DKD in this study was relatively similar to those obtained in Ethiopia and Tunisia, respectively $15.7 \%$ and $19.8 \%[15,16]$. In Tanzania, a prevalence of $24.7 \%$ has been found in 2013 [17]. This last one prevalence, as Mpondo et Al. said, may be attributable to the high prevalence of Schistosomiasis infection in the region of Tanzania, given its role in the etiology of renal disease [18-20].

According to the gender, DKD has been found positively associated with females, as reported in other studies [21, 22]. In a group of Indian living with diabetes, Verma et Al. reported that, the co-morbidity hypertension-DKD is prevalent at $45.4 \%$ [23]. However, in our study, we obtained a lower prevalence of this co- morbidity, at $8.4 \%$. The higher prevalence obtained in Indian was due to the inadequate definition of the DKD status by the authors, who did not take in consideration the recommendations of the ADA consensus on diabetes and CKD in the diagnostic and management of DKD [5].

According to the KDIGO consortium, CKD has been found prevalent at $59.6 \%$, staged from 1 to 5 [9]. This prevalence was high compared to the result reported by Choukem et Al. in 2012 in Yaounde, Cameroon, where a prevalence of $31 \%$ was obtained [24]. Differences happened between Choukem's study with ours, may be due to Cockcroft-Gault equation used as the methods to estimate the GFR there. Authors have reported the character of this equation to underestimate normal and high GFR compared to MDRD [25-27].

In this study, we diagnosed DKD according to the presence of persistent macro-albuminuria and microalbuminuria associated with retinopathy. Micro-albuminuria has been assessed in the diabetic population in Cameroon previously with a prevalence of 53.1\% in Yaounde (1999) [28], and 34.6\% in Bamenda in 2017 [29]. In agreement with these reports, the prevalence of micro-albuminuria found in our study confirms that microalbuminuria is frequent among diabetics in Cameroon. Microalbuminuria, as an important risk factor for cardiovascular disease, and facilitator of the progression of diabetes to renal impairment, has to be integrated during routine investigation of diabetes complications in Cameroon [30,31].

In this study, we used creatinine clearance alone, to estimate the GFR, according to the KDIGO guideline on CKD. However, a decreased eGFR is confirmed by an alternative filtration marker, cystatin $\mathrm{C}$, to estimate another GFR. Nevertheless, the careful respect of some important guides of KDOQI guideline on DKD has facilitated the identification of DKD cases.

\section{Conclusions}

The study showed that the prevalence of DKD is $15.2 \%$ and women were more exposed to develop it compared to men. Micro-albuminuria has been also shown to be more prevalent in diabetic population. The prevalence of CKD in diabetic population is high. These findings showed that, diabetic complications remain a serious problem and more actions should be taken to improve on the management of diabetes in Cameroon.

\section{Data Availability}

The original data used to support the findings of this study will be provided upon request.

\section{Conflict of Interests}

All the authors do not have any possible conflicts of interest.

\section{Funding}

We thank the Directors of the Medical Research and Applied Biochemistry Laboratory, and of the Regional Hospital of Buea and Ngaoundere for granting us laboratory space and reagents for the realization of this work. 


\section{Acknowledgements}

We are grateful to all patients who accepted to participate in this study. We want to thanks the Coordinator of the Lambe Foundation of Diabetes in Buea, the Director and his staff of the Regional Hospital of Buea and, the Director and his staff of the Regional Hospital of Ngaoundere for their contributions during this study. We are also grateful to the collaborators of the Medical Research and Applied Biochemistry Laboratory were samples were analyzed, for their contributions.

\section{References}

[1] O. Gheith, N. Farouk, N. Nampoory, M.. Halim and T. AlOtaibi, "Diabetic kidney disease: worldwide difference of prevalence and risk factors," Journal of Nephropharmacology, vol. 5, no. 1, pp. 49-56, 2016.

[2] Y. C. Lin, Chang, S. Y. Yang, K. D. u and T. S. Chu, "Update of pathophysiology and management of diabetic kidney disease," Journal of Formos and Medical Association, vol. 117, pp. 662-675, 2018.

[3] P. Fioretto, M. L. Caramori, M. Mauer, "The kidney in diabetes: Dynamic pathways of injury and repair. The Camillo Golgi Lecture 2007," Diabetologia, vol. 51, pp. 1347-1355, 2008.

[4] H. J. Anders, T. B. Nuber, B. Isermann and M. Schiffer, "CKD in diabetes: Diabetic kidney disease versus nondiabetic kidney disease," Nature Revue of Nephrology, vol. 14, pp. 361-377, 2018.

[5] K. R. Tuttle, G. L. Bakris, R. W. Bilous, J. L. Chiang, I. H. de Boer, J. Goldstein-Fuchs, I. B. Hirsch, K. Kalantar-Zadeh, A. S. Narva, S. D. Navaneethan, J. J. Neumiller, U. D. Patel, R. E. Ratner, A. T. Whaley-Connell and M. E. Molitch, "Diabetic Kidney Disease: A Report from an ADA Consensus Conference," Diabetes Care, vol. 37, pp. 2864-2883, 2014.

[6] I. H. de Boer, T. C. Rue, Y. N. Hall, P. J. Heagerty, N. S. Weiss, J. Himmelfarb, Temporal trends in the prevalence of diabetic kidney disease in the United States. Journal of American Medical Association, vol. 305, pp. 2532-2539, 2011.

[7] K. Al-Rubeaan, A. M. Youssef, S. N. Subhani, N. A. Ahmad, A. H. Al-Sharqawi, H. M. Al-Mutlaq, K. D. Satish, D. AlNaqeb, "Diabetic Nephropathy and Its Risk Factors in a Society with a Type 2 Diabetes Epidemic: A Saudi National Diabetes Registry-Based Study," PLoS ONE, vol. 9, no. 2, pp. e88956, 2014.

[8] W. H. Herman, R. E. Aubert, M. M. Engelgau, T. J. Thompson, M. A. Ali, E. S. Sous, M. Hegazy, A. Badran, S. J. Kenny, E. W. Gunter, A. M. Malarcher, R. J. Brechner, S. F. Wetterhall, F. DeStefano, P. J. Smith, M. Habib, S. Abd El Shakour, A. S. Ibrahim, E. M. El Behairy, "Diabetes Mellitus in Egypt: Diabetes Mellitus in Egypt: glycaemic control and microvascular and neuropathic complications," Diabetes Medicine, vol. 15, no. 12, pp. 1045-1051, 1998.

[9] A. S. Levey, P. E. de Jong, J. Coresh, M. El Nahas, B. C. Astor, K. Matsushita, R. T. Gansevoort, B. L. Kasiske and K. U. Eckardt, "The definition, classification, and prognosis of chronic kidney disease: a KDIGO Controversies Conference report," Kidney International, vol. 80, pp. 17-28, 2011.
[10] National Kidney Foundation, "KDOQI Clinical Practice Guideline for Diabetes and CKD: 2012 update," American Journal of Kidney Disease, vol. 60, no. 5, pp. 850-886, 2012.

[11] V. M. Muthuppalaniappan and M. M. Yaqoob. "Ethnic/Race Diversity and Diabetic Kidney Disease," Journal of Clinical Medicine. Vol. 4, pp. 1561-1565, 2015.

[12] K. A. Earl, K. K. Porter, J. Ostberg and J. S. Yudkin, "Variation in the progression of diabetic nephropathy according to racial origin," Nephrology and Dialysis Transplantation, vol. 16. Pp. 286-290, 2001.

[13] Centers for Disease Control and Prevention (CDC), "Report CMaMW. Prevalence of overweight and obesity among adults with diagnosed diabetes - United States, 1988-1994 and 19992002," MMWR Morbidity and Mortality Wkly Report, vol. 53, no. 45, pp. 1066-1068, 2004.

[14] Centers for Disease Control and Prevention. "National Diabetes Statistics Report, 2017. Atlanta, GA: Centers for Disease Control and Prevention," U. S. Department of Health and Human Services; 2017.

[15] D. Worku, L. Hamza and K. Woldemichael "Patterns of Diabetic Complications at Jimma University Specialized Hospital, Southwest Ethiopia". Ethiopian Journal of Health Science, vol. 20, no. 1, pp. 33-39, 2010.

[16] C. Bouzid, H. Smida, A. Kacem, Z. Turki, L. B. Salem, C. B. Rayana and C. B. Slama, "Kidney Failure in Tunisian Type 2 Diabetes Patients Hospitalized: Frequency and Associated Factors," La tunisie Medicale, vol. 89, no. 01, pp. 10-15, 2011.

[17] M. N. Janmohamed, S. E. Kalluvya, A. Mueller, R. Kabangila, L. R. Smart, J. A. Downs, et al., "Prevalence of chronic kidney disease in diabetic adult out-patients in Tanzania," $B M C$ Nephrology, vol. 14, pp. 183-187, 2013.

[18] B. C. T. Mpondo, E. Neilson, A. Ernest, "Prevalence of chronic kidney disease in diabetic adult out-patients in Tanzania," BMC Nephrology, vol. 17, pp. 71-72, 2016.

[19] M. A. Sobh, F. E. Moustafa, R. M. Ramzy, A. M. Deelder, M. A. Ghoneim, "Schistosoma haematobium-induced glomerular disease: an experimental study in the golden hamster," Nephron, vol. 57, pp. 216-24, 1991.

[20] W. L. C. dos-Santos, G. M. M. Sweet, M. Bahiense-Oliveira, P. N. Rocha, "Schistosomal glomerulopathy and changes in the distribution of histological patterns of glomerular diseases in Bahia, Brazil," Mem Inst Oswaldo Cruz, vol. 106, pp. 901904, 2011.

[21] M. K. Yu, C. R. Lyles, L. A. Bent-Swah, B. A. Young and the Pathways authors, "Risk factor, age and sex differences in chronic kidney disease prevalence in a diabetic cohort: The Pathways Study," American Journal of Nephrology, vol. 36, no. 3, pp. 245-251, 2012.

[22] I. Laranjinhaa, P. Matiasa, S. Mateusb, F. Aguiarb, P. Pereirab, M. P. Santosb, R. Costab, A. Lourencob, J. Guiab, J. D. Barataa and B. L. Campos, "Diabetic kidney disease: Isthereanon-albuminuric phenotype in type 2 diabetic patients?" Nefrologia, vol. 36, no. 5, pp. 503-509, 2016.

[23] A. Verma, S. Vyas, A. Agarwal, S. Abbas, D. P. Agarwal and R. Kumar, "Diabetic Kidney Disease and Hypertension: A True Love Story," Journal of Clinical Diagnosis and Research, vol. 10, no. 3, pp. 11-13, 2016. 
[24] S. P. Choukem, A. Dzudi, M. Dehayem, M. P. Halle, M. S. Doualla, H. Luma and P. Kengne, "Comparison of different blood pressure indices for the prediction of prevalent diabetic nephropathy in a sub-Saharan African population with type 2 diabetes," Pan-African Medical Journal, vol. 11, pp. 67-74, 2012 .

[25] V. Rigalleau, M. C. Beauvieux, C. Gonzalez, C. Raffaitin, C. Lasseur, C. Combe, P. Chaveau, R. De la Faille, C. Rigothier, N. Barthe and H. Gin, "Estimation of renal function in patients with diabetes," Diabetes Metabolic, vol. 37, pp. 359-366, 2011.

[26] D. D. Agoons, E. V. Balti, F. F. Kaze, M. Azabji-Kenfack, G. Ashungtantang, A. P. Kegne, E. Sobngwi and J. C. Mbanya, "Performance of three glomerular filtration rate estimation equations in a population of sub-Saharan Africans with Type 2 diabetes," Diabetes Medical, vol. 33, no. 9, pp. 1291-1298, 2016.

[27] M. K. I. Mbarawa, G. M. Ndoe, L. L. Mohamadou, T. T. Marcel, S. L. A. Kagoue and G. D. Fokam, "A Comparison of three Methods to Estimate the Glomerular Filtration Rate in
Diabetic Patients at the Ngaoundere Regional Hospital (Cameroon)," International Journal of Health and Biological Science, vol. 2, no. 1 pp. 6-10, 2019.

[28] E. Sobngwi, J. C. Mbanya, E. N. Moukouri and K. B. Ngu, "Microalbuminuria and retinopathy in a diabetic population of Cameroon," Diabetes Research and Clinical Practice, vol. 44, no. 3, pp. 191-196, 1999.

[29] M. E. A. Bissong, G. N. Teke, M. Sanje, H. Goneh, F. E. T. Foka and H. L. Kamga, "Microalbuminuria in Diabetic Patients in the Bamenda Health District,". Scientific Journal of Clinical Medicine, vol. 6, no. 4, pp. 63-67, 2017.

[30] S. C. Satchell and J. E. Tooke, "What is the mechanism of microalbuminuria in diabetes: a role for the glomerular endothelium?" Diabetologia, vol. 51, no. 5, pp. 714-725, 2008.

[31] E. T. Efundem, J. C. N. Assob, V. F. Feteh and S. P. Choukem, "Prevalence and associations of microalbuminuria in proteinuria-negative patients with type 2 diabetes in two regional hospitals in Cameroon: a cross-sectional study," $B M C$ Research Notes, vol. 10, pp. 477-481, 2017. 